Arthropod phylogeny

Arthropod Phylogeny: With Special Reference to Insects. By H.B. Boudreaux. Pp. 320. (Wiley: New York and Chichester, UK, 1979.) £15.

THE past fifteen years have seen the publication of major works on arthropod phylogeny by Sharov, Hennig, Anderson and, notably, by Manton. Professor Boudreaux has now added to them a book distinguished by two guiding principles. Firstly, he believes that the Arthropoda form a single monophyletic taxon and therefore sets himself firmly against the recent tendency to regard them as three or more lineages, each independently evolved from primitive lobopod ancestors. Secondly, as an advocate of Hennig's methods of phylogenetic reconstruction, he proceeds by defining monophyletic sister-groups strictly through their common specialisations and abandoning, without much hesitation, the paraphyletic and polyphyletic taxa of the past.

After treating the Onychophora as a phylum separate from but related to the Arthropoda, Boudreaux devotes almost a third of his book to the affinities of the arthropod classes and the remaining twothirds to the mutual relationships of the insect orders. The great diversity of the phylum, the volume of factual material, and the closely reasoned interpretations defy a brief summary, but the book has the great merit of presenting explicitly the foundations (mainly more or less familiar morphological data from living forms) on which the inevitably somewhat speculative inferences are based. Essentially the author recognises two main branches in arthropod evolution: a Cheliceromorph line, with the Pycnogonids, Xiphosurids, Eurypterids and Arachnids as its major subdivisions, and a more varied Gnathomorph line. The latter includes the Trilobites and the Mandibulata which (pace Manton) comprises the Crustacea, Myriapoda and the monophyletic Insecta.

To express his phylogenetic views in taxonomic terms, Boudreaux does not flinch from introducing, between the class Insecta and its constituent orders, no fewer than thirteen different taxonomic categories and the appropriate taxa, each duly named and defined. Within this rather cumbersome framework the evolutionary affinities of the orders are discussed in ways that are sometimes widely acceptable, sometimes more controversial. The Apterygota, Palaeoptera and Exopterygota are regarded as paraphyletic and discarded, although the Entognathan apterygotes are treated as a natural group. The order Mallophaga is restricted to the Amblycera, while a sister-group, the Anoplura, is redefined to accommodate the Ischnocera, Rhynchophthirina and Siphunculata. The Plecoptera, Dermaptera and Embioptera, all with notoriously obscure affinities, are hardly more convincingly placed than in alternative classifications, and similar doubts might also apply to the Thysanoptera, Strepsiptera and Siphonaptera. The Sialoidea, Raphidioidea and Plannipennian Neuroptera all receive separate ordinal status, but the Boreidae and Micropterigidae are denied it.

In many respects the resulting scheme for the insects is not unlike those proposed by Hennig or Kristensen. What must strike a more sceptical reader, however, is the tantalising scarcity of really crucial evidence and fully convincing arguments.

\section{Semiconductor devices}

Semiconductors and Electronic Devices. By A. Barl-Lev. Pp. 381. (Prentice-Hall International: London and Englewood Cliffs, New Jersey, 1979.) Hardback £11.95, \$22.95; paperback £8.95, \$18.95.

THIS medium-sized book written by an electrical engineer for electrical engineers embarks on the ambitious task of introducing the reader to the physical principles of semiconductor materials and leading him on to a more detailed discussion of various aspects of junction and, to a lesser extent applications of semiconductor devices. In a sense, the title is slightly misleading, implying that devices other than semiconductor ones are being treated while, in fact, the discussion of vacuum devices is minimal.

The discussion of the physical and technological principles relating to semiconductor materials covers 114 pages in 8 chapters and is necessarily sketchy, as the author admits in the preface. However, I accept that the treatment is adequate for an electrical engineering student wishing to concentrate on the practical aspects of device performance.

The next three chapters are devoted to a discussion of various aspects of junction diodes, beginning with their static characteristics and including a description of varactor diodes. The optoelectronic devices mentioned include the PIN detectors, solar cells, light emitting diodes, optic couplers and even injection lasers the treatment here is possibly at its most sketchy in view of the different physical background required for full understanding. A more thorough chapter on the dynamic response gives a discussion of diode switching.

The treatment of transistors begins interestingly and unconventionally with a discussion of field effect transistors both of the junction and of the insulated gate
Despite his confident style the author does, in fact, admit the tentative nature of many phylogenetic hypotheses. Their importance lies less, perhaps, in the permanent value of the conclusions reached than in their immediate heuristic value. In this sense the views and arguments of Boudreaux and those he represents should be welcomed: they will, along with the theories of their opponents, promote the study of Arthropod morphology, development and classification, and so, one hopes, make possible one day a more widely agreed phylogeny of the Arthropoda.

R.G. Davies

R.G. Davies is Reader in Entomology at Imperial College, University of London, UK.

types, with a brief reference to integrated circuit design. This is followed by a chapter on the amplifying stage, transistor model and the equivalent circuit which is based on the characteristics of the MOS transistor but includes mention of vacuum device and bipolar transistor characteristics, neither of which had yet been introduced. The discussion of bipolar transistors starts with the large signal model and maximum ratings and only then proceeds to small-signal low- and highfrequency models. This is a sensible arrangement and these two chapters show a rather deeper level of treatment than most of the others. One chapter deals with an introduction to integrated circuits, both bipolar and MOS, to charge-coupled devices and integrated injection logic.

The chapter on microwave devices describes very briefly the relevant contructions of the bipolar and junction FETs, gives a very cursory discussion of varactor, step-recovery and IMPATT diodes, and finishes with the transferred electron devices. The main body of the text is completed with a chapter on siliconcontrolled rectifiers and triacs. There are three appendices on two-port representations, thermionic emission and vacuum tubes - the last two somewhat incongruous in the present context.

On the whole, as an introductory text for electrical engineers this is a good and interesting book. It mentions, if only briefly, most of the recent developments in semiconductor devices that the student is likely to come across. There are useful Problems at the end of each chapter. My general criticism of this strongly deviceoriented text is the relative paucity of actual device characteristics - the student would have benefitted a great deal from being able to see more performance data. Also, the list of 22 references for further reading is not adequate to guide a reader who wishes to follow up specialised topics.

A.K. Jonscher

A.K. Jonscher is Professor of Solid-State Electronics at Chelsea College, University of London, UK. 